University of New Hampshire

University of New Hampshire Scholars' Repository

Space Science Center

Institute for the Study of Earth, Oceans, and

Space (EOS)

1994

\title{
COMPTEL detection of the variable radio source GT $0236+610$
}

\author{
R VanDijk \\ ESTEC \\ H Bloemen \\ Space Research Organization of the Netherlands \\ W Hermsen \\ Space Research Organization of the Netherlands (SRON) \\ W Collmar \\ Max-Planck-Institut für extraterrestriche Physik \\ R Diehl \\ Max-Planck-Institut für extraterrestriche
}

See next page for additional authors

Follow this and additional works at: https://scholars.unh.edu/ssc

Part of the Astrophysics and Astronomy Commons

\section{Recommended Citation}

COMPTEL detection of the variable radio source GT 0236+610 van Dijk, R. and Bloemen, $\mathrm{H}$. and Hermsen, W. and Collmar, W. and Diehl, R. and Lichti, G. G. and Schönfelder, V. and Steinle, H. and Strong, A. and Bennett, K. and Much, R. and McConnell, M. and Connors, A., AIP Conference Proceedings, 304, 324-328 (1994), DOl:http://dx.doi.org/10.1063/1.45581

This Conference Proceeding is brought to you for free and open access by the Institute for the Study of Earth, Oceans, and Space (EOS) at University of New Hampshire Scholars' Repository. It has been accepted for inclusion in Space Science Center by an authorized administrator of University of New Hampshire Scholars' Repository. For more information, please contact Scholarly.Communication@unh.edu. 


\section{Authors}

R VanDijk, H Bloemen, W Hermsen, W Collmar, R Diehl, G G. Lichti, V Schonfelder, H Steinle, A W. Strong, K Bennett, M Much, Mark L. McConnell, and A Connors 


\section{AIP $\mid$ proceedings}

\section{COMPTEL detection of the variable radio source GT $0236+610$}

R. van Dijk, H. Bloemen, W. Hermsen, W. Collmar, R. Diehl, G. G. Lichti, V.

Schönfelder, H. Steinle, A. Strong, K. Bennett, R. Much, M. McConnell, and A.

Connors

Citation: AIP Conference Proceedings 304, 324 (1994); doi: 10.1063/1.45581

View online: http://dx.doi.org/10.1063/1.45581

View Table of Contents:

http://scitation.aip.org/content/aip/proceeding/aipcp/304?ver=pdfcov

Published by the AIP Publishing

\section{Articles you may be interested in}

Comptel observations of the quasar PKS $0528+134$

AIP Conf. Proc. 304, 659 (1994); 10.1063/1.45668

EGRET observations of Mrk 421 in Phase 1 and Phase 2 of the Compton Observatory's viewing program-A summary

AIP Conf. Proc. 304, 582 (1994); 10.1063/1.45656

COMPTEL upper limits for Seyfert galaxies

AIP Conf. Proc. 304, 556 (1994); 10.1063/1.45652

A transient $\mathrm{MeV}$ range gammaray source observed by HEAO-1

AIP Conf. Proc. 304, 255 (1994); 10.1063/1.45628

$\mathrm{MeV}$ emission from the blackhole candidate GRO J0422+32 measured with COMPTEL

AIP Conf. Proc. 304, 197 (1994); 10.1063/1.45619 


\title{
COMPTEL DETECTION OF THE VARIABLE RADIO SOURCE GT $0236+610$
}

\author{
R. van Dijk ${ }^{1}$, H. Bloemen, W. Hermsen
}

SRON-Leiden, P.O. Box 9504, NL-2300 RA Leiden, The Netherlands

W. Collmar, R. Diehl, G.G. Lichti, V. Schönfelder, H. Steinle, A. Strong

Max-Planck Institute für Extraterrestrische Physik, P.O. Box 1603, 85740 Garching, F.R.G.

K. Bennett, R. Much

Astrophysics Division, ESTEC, NL-2200 AG Noordwijk, The Netherlands

M. McConnell, A. Connors

Space Science Center, Univ. of New Hampshire, Durham NH 03824, U.S.A.

\section{ABSTRACT}

The highly variable and non-thermal radio source GT $0236+610$ exhibits outbursts with a period of 26.496 days, modulated by a four-year period. Recent EGRET observations confirmed that this source is the counterpart of the COS-B source 2CG $135+01$.

COMPTEL observed this source during three observations in Phase I. We report here on the detections in each of the observations, the time-averaged spectrum, and address the question of time variability with respect to the radio phase.

\section{INTRODUCTION}

The $\gamma$-ray source $2 \mathrm{CG} 135+01$ was one of the unidentified strong sources in the COS-B catalogue (Hermsen et al. 1977; Swanenburg et al. 1981). Soon after the discovery, two possible counterparts were proposed. One of these was the luminous low-redshift QSO 4U0241+61, for which the Uhuru and SAS 3 error boxes overlap the COS-B error box (Apparao et al. 1978). The other counterpart proposed was the highly variable, non-thermal radio source GT 0236+610 (Gregory \& Taylor 1978). Recent observations of 2CG 135+01 with EGRET, have improved its location error box, and exclude the QSO from being the counterpart (von Montigny et al. 1993).

GT $0236+610$, coincident with the B0 Ve star $\mathrm{LSI}+61^{\circ} 303$, was shown to exhibit non-thermal radio outbursts with a period of 26.496 days (Taylor \& Gregory 1982). These outbursts are characterised by a short ( $\sim$ few days) risetime, followed by a somewhat slower decline. During the onset of the outbursts, the spectral index $\alpha\left(S_{\nu} \propto \nu^{\alpha}\right)$ decreases with increasing radio flux, and reaches a minimum at maximum radio flux (Taylor \& Gregory 1984). The radio outbursts are modulated with a four-year period (Gregory et al. 1989). The 26.496 day period has also been detected in the optical (Mendelson \& Mazeh 1989), but not in the IR (D'Amico et al. 1987), UV (Howarth 1983), or X-rays (Bignami et al. 1981).

The current theoretical picture of 2 CG $135+01 /$ GT $0236+610 / \mathrm{LSI}+61^{\circ} 303$ is that of a binary system consisting of a B0 Ve star and a compact object, with a 26.496 day orbital period. Spectroscopic observations indicate that, for an inclination $i=70^{\circ}-80^{\circ}$ and a $5 \mathrm{M}_{\odot}-10 \mathrm{M}_{\odot}$ star, the mass of the compact object is in the range $1.1 \mathrm{M}_{\odot}-1.5 \mathrm{M}_{\odot}$.

\footnotetext{
${ }^{1}$ Also Astronomical Institute, University of Amsterdam, Kruislaan 403, NL-1098 SJ Amsterdam, The Netherlands
} 
Based on this, Maraschi \& Treves (1981) proposed the so called young pulsar model. In this model, it is assumed that the compact object is a young pulsar (age $10^{4}-10^{5} \mathrm{yr}$ ), with a strong relativistic wind. The $\gamma$-ray emission is thought to originate in the shock front between the pulsar wind and the stellar wind, through inverse-Compton scattering of the primary's optical photons off the relativistic electrons. These electrons also produce the (periodic) radio emission through synchrotron radiation. Because the orbit is eccentric, the magnetic field at the shock is higher during periastron passage, such that the resulting larger accelerations of the electrons lead to the observed periodicity. Another model, the supercritical accretion model, was proposed by Taylor \& Gregory $(1982 ; 1984)$. In this model, the outbursts are caused by luminosity-driven shocks as a result of supercritical accretion. The required large $\dot{M}$ for this process is likely caused by Roche-lobe overflow of the primary.

\section{INSTRUMENT AND OBSERVATIONS}

The COMPTEL instrument aboard CGRO, is sensitive to $\gamma$-rays between $0.75 \mathrm{MeV}$ and $30 \mathrm{MeV}$, and has a field of view of $\sim 1$ steradian. The energy resolution is $5-10 \%$, and the positional accuracy is typically $1^{\circ}$. The detection mechanism is based on Compton scattering, the dominant process in this energy range. Incoming photons are ideally first Compton scattered in the upper detector, and subsequently totally absorbed in the lower detector. In practice, however, the absorption in the lower detector is not always complete. This, and other effects, complicate the response function. For a complete description of COMPTEL, and its characteristics, the reader is referred to Schönfelder et al. (1993).

Table I. List of COMPTEL observations during 1991 - 1992 of GT 0236+610 (in CGRO notation). The columns list: 1) the start time, 2) the end time, 3) the corresponding radio-phase intervals (see Gregory et al. 1989) and 4) the angular distance $\zeta$ to the pointing direction.

\begin{tabular}{ccccc}
\hline Obs. & $\begin{array}{c}\text { Start } \\
U T\end{array}$ & $\begin{array}{c}\text { End } \\
U T\end{array}$ & Radio Phase & $\begin{array}{c}\zeta \\
{\left[{ }^{\circ}\right]}\end{array}$ \\
\hline 15.0 & Nov. $28,12: 41$ & Dec. 12, 16:42 & {$[0.096-0.631]$} & 22.7 \\
31.0 & Jun. 11, 16:11 & Jun. 25, 13:17 & {$[0.499-0.023]$} & 29.9 \\
34.0 & Jul. 16, 16:40 & Aug. 06, 14:38 & {$[0.820-0.610]$} & 26.5 \\
\hline
\end{tabular}

COMPTEL completed a sky survey during the observations of Phase I (May 1991 November 1992). GT $0236+610$ was within $30^{\circ}$ of the pointing in three Phase I observations: Obs. $15.0,31.0$ and 34.0 . Table 1 lists the observation periods, the corresponding radio phases and the angular distance $\zeta$ to the pointing direction. During Obs. 31.0 and 34.0 only real-time telemetry existed. Due to incomplete telemetry coverage, the effective exposures are less than expected based on the durations of these observations.

According to Taylor et al. (1992), the radio-outburst patterns differ drastically between cycles. On the average, however, the peak flux occurs at phase $0.6 \pm 0.1$. Obs. 31.0 therefore has the best coverage of the radio-outburst phase interval (see also Fig. 3). 


\section{ANALYSIS}

Each of the three observations was analysed in four energy ranges: $0.75-1 \mathrm{MeV}, 1-3$ $\mathrm{MeV}, 3-10 \mathrm{MeV}$ and 10-30 MeV. In addition, the data of the different observations were added and analysed together. Location maps were produced using a maximum-likelihood method, which is also used to determine the fluxes. A full description of this method can be found elsewhere (de Boer et al. 1992; Bloemen et al. 1993). Note that the point-spread function of the COMPTEL instrument is energy dependent. Assuming a certain spectrum (e.g. power law, Wien), the exact spectral shape (e.g. spectral index, temperature) must be found iteratively by varying the relevant parameters. Convergence is reached when the ratio of the fluxes in different energy ranges is consistent with the assumed spectral shape.

\section{RESULTS}

We have detected GT $0236+610$ in all three observations. The detection significance in the sum of the three observations in Table 1 is $\sim 5.5 \sigma(1-30 \mathrm{MeV})$. In the single observations, GT $0236+610$ is detected only below $3 \mathrm{MeV}$ with significances $\sim 3.0 \sigma, \sim 3.5 \sigma$ and $\sim 2 \sigma$ respectively. Fig. 1 shows the $95 \%$ and $99 \%$ error location contours for the sum of the three observations of Table 1 (1-30 MeV). The position of GT $0236+610$ is denoted by the asterisk. Note that, although the error box of COMPTEL is relatively large, the EGRET observations exclude the QSO 4 U0241+61 from being the counterpart (von Montigny et al. 1993).

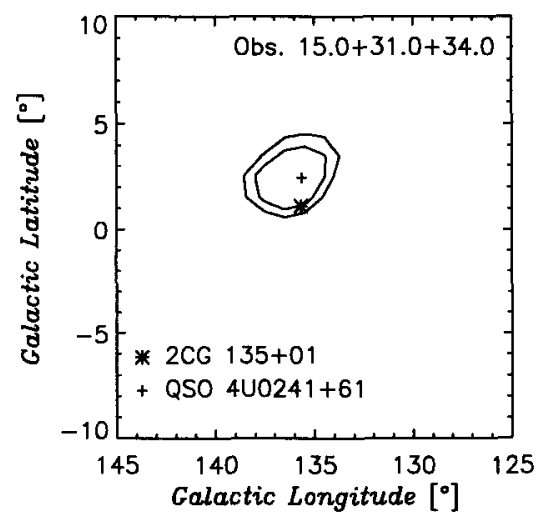

Fig 1. Location map for the sum of the observations 15.0,31.0 and $34.0(1-30 \mathrm{MeV})$. The contour levels are the $95 \%$ and $99 \%$ error location contours. The pasition of GT $0236+610$ is denoted by the asterisk. For completeness, the position of the QSO $4 \mathrm{U} 0241+61$ is also indicated.

For the analysis presented here, a power law was assumed as input spectrum. From the flux values derived for power laws with different spectral indices, we find that the COMPTEL flux points are consistent with a power-law spectrum with spectral index $\sim 1.7$. This spectrum is thus softer than the spectrum measured at energies $<1 \mathrm{MeV}(\alpha \approx 1.0$, Perotti et al. 1980), but not as soft as the spectrum measured at higher energies $\left(\alpha=2.0_{-0.25}^{+0.40}\right.$, Swanenburg et al. 1981). Fig. 2 shows the COMPTEL spectral points, together with the results from the MISO and COS-B instruments. 


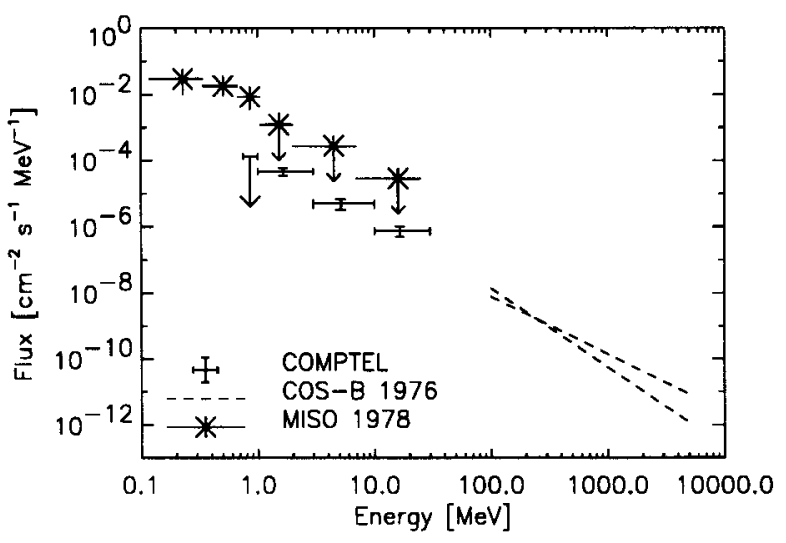

Fig 2. The COMPTEL data points and $2 \sigma$ upper limit for the time-averaged spectrum of observations 15.0, 31.0 and 34.0. Also plotted are the MISO flux points (Perotti et al. 1980) and the COS-B $>100 \mathrm{MeV}$ flux represented by two power laws with spectral indices 1.75 and 2.4 (Swanenburg et al. 1981).

\section{TIME VARIABILITY?}

Since a 26.496 day periodicity has been detected at optical and radio wavelengths, it is interesting to see whether the three COMPTEL observations show any hint for time variations. Because each observation has a different coverage of the radio outburst phase interval, time variations might be visible if the flux variation with phase is strong enough.

A simple comparison of the fluxes in the 1-3 MeV energy range (for which the most significant results were obtained in each of the three observations), does not reveal significant deviations from a constant flux. This is shown in Fig. 3, where the 1-3 MeV fluxes for the complete observations have been plotted against the coverage of the radio outbursts phase interval. This implies that any time variations present in the $\mathrm{MeV}$ emission must be small. In order to place more stringent limits, the observations will be binned according to phase, and each phase interval will be analysed separately (van Dijk et al. 1994).

\section{SUMMARY}

COMPTEL observed the binary GT $0236+610$ three times in Phase I. In two observations a $\sim 3.5 \sigma$ detection is obtained. The detection significance for the sum of the observations is $\sim 5.5 \sigma(1-30 \mathrm{MeV})$. The spectral points measured by COMPTEL are consistent with a power law of spectral index $\sim 1.7$. Between the three observations, no significant time variability is detected. 


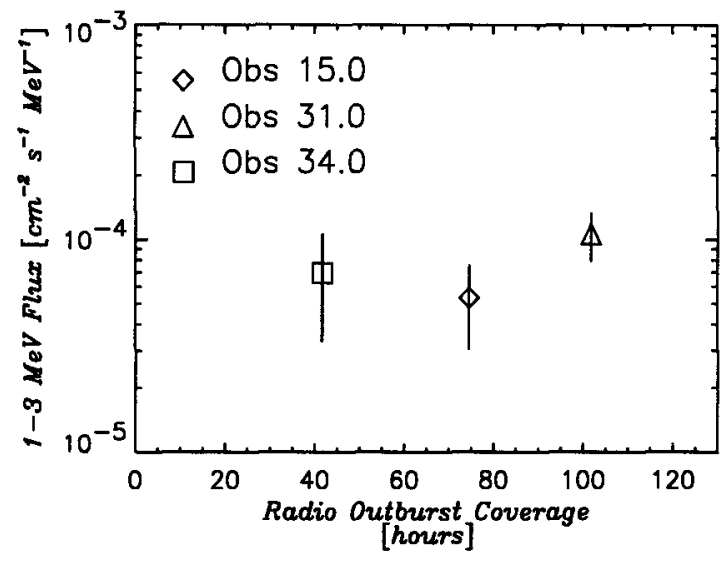

Fig 3. The COMPTEL 1-3 MeV flux points plotted against the coverage of the radio-outburst phase interval 0.5-0.7.

\section{REFERENCES}

Apparao K.M.V. et al., 1978, Nat 273, 450

Bignami G.F. et al., 1981, ApJ 247, L85

Bloemen H. et al., 1993, ApJS, in press D'Amico N., 1987, A\&A 180, 114

de Boer H. et al., 1992, in: Data Analysis in Astronomy IV, eds. V. Di Gesù (New York:

Plenum Press), Vol 59, p241

Gregory P.C., Taylor A.R., 1978, Nat 272, 704

Gregory P.C. et al., 1989, ApJ 339, 1054

Hermsen W. et al., 1977, Nat 269, 494

Howarth I.D., 1983, MNRAS 203, 801

Maraschi L., Treves A., 1981, MNRAS 194, 1P

Mendelson H., Mazeh T., 1989, MNRAS 239, 733

Perotti F. et al., 1980, ApJ 239, L49

Schönfelder et al., 1993, ApJS 86, 657

Swanenburg B.N. et al., 1981, ApJ 243, L69

Taylor A.R., Gregory P.C., 1982, ApJ 255, 210

Taylor A.R., Gregory P.C., 1984, ApJ 283, 273

Taylor A.R. et al., 1992, ApJ 395, 268

van Dijk R. et al., 1994, A\&A, in preparation

von Montigny C. et al., 1993, IAU Circ. 5708 